\begin{tabular}{lllllllllllllllllllllllllllllllll}
\hline$R$ & $E$ & $V$ & I & S & T & A & D & E & E & S & T & U & D & I & O & S & I & N & T & E & R & N & A & C & I & O & N & A & L & E & S
\end{tabular}

\title{
La violencia sexual contra la mujer
}

\section{Legalización de los sistemas de protección*}

\author{
Tatiana Rein Venegas
}

Partiendo de la premisa de que la protección otorgada a la mujer contra la violencia sexual puede variar según la oportunidad en que se produce el atentado, se examinan los sistemas internacionales de protección de los derechos humanos aplicables específicamente a la materia, con especial referencia al sistema de las Naciones Unidas y al Sistema Interamericano que se hacen efectivos en períodos de paz, así como al sistema que se aplica en épocas de conflicto armado, en este, centrándose en la Corte Penal Internacional y al Estatuto pertinente. El estudio se basa en la teoría de la legalización, según se expone en la obra Legalization and World Politics, que establece los requisitos que debe reunir la institucionalidad para determinar su grado de desarrollo.

De acuerdo con la aplicación de cada uno de los aspectos de la legalización -obligación, precisión y delegación- a los sistemas de protección elegidos, puede afirmarse que, a diferencia de otros, el sistema de las Naciones Unidas tiene escaso nivel de desarrollo. Sin embargo, los índices de violencia son similares en todos ellos, lo que dificulta la aplicación directa de los efectos de la teoría a esta materia o al menos pone de manifiesto sus complejidades, lo que obliga a adoptar diversas medidas en relación con cada una de las causas de esta particular violencia.

\section{INTRODUCCIÓN}

sí como resulta inquietante la tardía adopción de normas internacionales para proteger a la mujer, de la violencia sexual a que puede verse sometida, también llama la atención que dicho tratamiento internacional sea disímil y dependa del momento en que se produce el ataque, esto es, si ocurre en períodos de paz o de conflicto armado.

Para determinar la veracidad de esta

* Síntesis de la tesis para optar al grado de Magíster en Estudios Internacionales de la Universidad de Chile, junio de 2005. 
afirmación, se examinarán los mecanismos internacionales existentes para la protección de la mujer de la violencia sexual y su diferente tratamiento, según el período en que tenga lugar el hecho.

\section{Las instituciones que operan en épocas de conflicto armado protegen mejor a la mujer.}

La obra Legalization and World Politics (Legalización y Política Mundial), relativa a la legalización de las instituciones, es un marco apropiado para realizar este análisis, que postula requisitos copulativos que se deben exigir a la institucionalidad para determinar su grado de desarrollo.

La aplicación de este enfoque a las instituciones que protegen a la mujer de la violencia sexual permitirá comprobar el nivel alcanzado, tanto por aquellas que operan en períodos de paz como por aquellas que lo hacen en época de conflicto armado, así como determinar si la legislación vigente es adecuada o inadecuada. Al mismo tiempo, ayudará a establecer cuáles son sus aspectos más débiles, que es preciso reforzar para brindar la protección debida.

Las hipótesis en que se basa el trabajo son, primero, que los mecanismos internacionales de protección con que cuen- ta la mujer para enfrentar la violencia sexual de que puede ser objeto en tiempos de paz son insuficientes y, segundo, que las instituciones que operan en épocas de conflicto armado se encuentran más desarrolladas y brindan mayor protección a la mujer ante dichos abusos.

A lo largo del trabajo se intentará demostrar parte de estas afirmaciones, haciendo un recorrido por los sistemas de protección que operan en ambos períodos y evaluándolos desde la perspectiva de su legalización ${ }^{2}$ y así determinar el bajo nivel alcanzado por los primeros y el mayor desarrollo de los segundos.

\section{LA LEGALIZACIÓN DE LAS INSTITUCIONES}

El estudio contenido en la obra Legalization and World Politics resulta novedoso porque, si bien el uso de la ley en la política internacional ya se ha analizado desde diversos puntos de vista, no se habían incorporado los tres aspectos que proponen los autores en este enfo$q^{2} e^{3}$. De esta manera, se podrían imponer mayores exigencias a la institucionalidad, en este caso la institucionalidad internacional, en función de esos aspectos, que no solo se refieren a la existencia misma de regulación, sino a las características específicas que esta debe cumplir.

Goldstein, Judith, Miles Kahler, Robert O. Keohane, y Anne-Marie Slaughter (eds.), Legalization and World Politics, Cambridge, Massachusetts y Londres, The MIT Press, 2001.

2 El concepto de legalización es desarrollado en la obra a que se ha hecho referencia y será explicado en profundidad en el primer capítulo de esta tesis.

3 Goldstein, Judith et al., op. cit., pp. 4-.9. 
Además, resulta conveniente desarrollar la normativa vigente utilizando la fórmula aportada por los autores, debido a los efectos que le atribuyen a la legalización, cuando sostienen que esta puede modificar de manera permanente la naturaleza de las políticas tantos internas como transnacionales de los países participantes, que el derecho internacional se puede internalizar y que el acceso de actores internos a cortes o instituciones internacionales, que son terceros independientes, puede llevarlos a modificar sus expectativas y su comportamiento, y promover la expansión de la legalización ${ }^{4}$.

El argumento de que la legalización puede cambiar las políticas internas es precisamente la clave del estudio. Se pretende probar que un aumento de la legalización en el área de la violencia contra la mujer cambiaría la política de los Estados en los ámbitos judicial, administrativo, educacional, entre otros, y conduciría a modificar los patrones de conducta de sus nacionales, produciendo una disminución de la incidencia de este fenómeno.

Los autores sostienen que la ley está estrechamente relacionada con la política: afecta los procesos políticos y su resultado. La relación entre derecho y política es recíproca y se aprecia a través de las instituciones, que son un conjunto de reglas, normas y procedimientos de toma de decisiones que determinan expectativas, intereses y comportamientos de los actores ${ }^{5}$.
Para que las instituciones se encuentren legalizadas deben reunir una serie de requisitos, a saber:

\section{La legalidad es clave para la cooperación internacional.}

1. Obligatoriedad: los actores deben adecuar su comportamiento a las reglas y procedimientos y a lo dispuesto por el derecho internacional.

2. Precisión: las reglas deben definir claramente la conducta que mandan, prohíben o permiten.

3. Delegación: facultad de interpretación, monitoreo, implementación y resolución de controversias que se entrega a un tercero ${ }^{6}$.

Cada uno de los aspectos señalados puede variar desde el punto máximo, en que los requisitos se reúnen a la perfección, hasta el punto mínimo, en que no se da ninguno de ellos. Cada uno puede variar en forma independiente, permitiendo una amplia gama de combinaciones ${ }^{7}$.

Los autores señalan que una consecuencia clave de la legalización para la cooperación internacional son sus efectos en materia de cumplimiento de las obligaciones internacionales, que sin legalización son difíciles de medir y definir. ${ }^{8}$

De acuerdo con lo manifestado en la obra, al definir la legalización en términos de obligación, precisión y delegación, en-

\footnotetext{
4 Ibid., p. 15.

5 Ibid., p. 3.

6 Ibid., pp. 3 у 17.

7 Ibid., pp. 4-18.

$8 \quad$ Ibid., p. 13.
} 
frentamos dimensiones identificables de variaciones, entre las categorías que según los autores pueden adoptar, cuyos efectos en el comportamiento internacional pueden ser empíricamente explorados ${ }^{9}$. Bastará con comprobar la adecuación a dichos parámetros para esperar que se cumplan los efectos, los que además podrán ser medidos.

\section{Las limitaciones impuestas por las} normas más cercanas a tipos legales son superiores a las normas de cortesía y moralidad.

\section{Obligación}

La obligatoriedad se relaciona con la vinculación del comportamiento de los actores internacionales. Las limitaciones que impone cierta clase de normas o compromisos, en particular los que más se acercan a tipos legales, son muy superiores a las que producen, por ejemplo, las normas de cortesía o de moralidad. ${ }^{10}$

De acuerdo con el modelo propuesto, la obligatoriedad contempla diversas posibilidades: desde la obligación incondicional, en la que el lenguaje $u$ otro indicio dejan de manifiesto la intención de ser legalmente vinculante, pasando por los tratados de tipo político, en que las condiciones de obligatoriedad se encuentran más bien implícitas; la posibilidad de formular reservas a obligaciones específicas, así como de incorporar en el propio tratado cláusulas de escape; las exhortaciones a tener comportamientos determinados; las normas adoptadas sin intención de darles autoridad legal, es decir, simples recomendaciones, hasta el expreso rechazo a que sean vinculantes ${ }^{11}$.

\section{Precisión}

Este aspecto se refiere a la adopción de reglas determinadas, que establecen lo que se espera de los actores internacionales. Ellas acotan el alcance que puede tener la interpretación. Si se trata de un conjunto de reglas, no solo deben ser claras sino relacionarse entre sí de manera no contradictoria, creando un marco que sea coherente en caso de interpretación. En general, si las reglas son precisas ellas son altamente elaboradas, contienen condiciones y explicaciones detalladas y establecen los comportamientos prohibidos, entre otras condiciones ${ }^{12}$.

\section{Delegación}

Esta dimensión se traduce en la cesión de la facultad de hacer operativos los acuerdos que entregan los Estados u otros actores internacionales a cortes, árbitros $\mathrm{u}$ organismos administrativos. A estos organismos se les otorga la facultad de in-

\footnotetext{
Ibid., p. 19.

Ibid., p. 24.

Ibid., pp. 26-27.

Ibid., pp. 28-29.
} 
terpretar las normas, aplicarlas e incluso elaborar nuevas. Si los Estados acuerdan someterse a las decisiones de estas terceras partes, sobre la base de reglas claras y generalmente aplicables, el nivel de legalización será mayor. En cambio, si se contempla la negociación política entre las partes, que pueden aceptar o rechazar propuestas sin justificación legal, el nivel de legalización será menor ${ }^{13}$.

La independencia significa que los órganos de solución de controversias pueden decidir imparcialmente respecto de los intereses de los Estados.

La delegación no sólo se refiere a la resolución de controversias, sino que puede consistir en facultades consultivas, interpretativas y operacionales ${ }^{14}$.

En la delegación es posible distinguir tres aspectos, a saber, independencia, acceso e implementación. Los tres influirían en los diversos grados en que ella puede darse. La independencia consiste en la posibilidad de que los órganos de resolución de controversias decidan imparcialmente respecto de los intereses específicos de los Estados. El acceso se refiere a la facilidad con que las partes, que no sean Estados, pueden influir en la agenda del tribunal. La implementación ${ }^{15}$ determina el grado con que se pueden hacer operativas las decisiones adoptadas por el órgano, sin que los gobiernos deban adoptar acciones para ello ${ }^{16}$.

\section{LA LEGALIZACIÓN DE LOS SISTEMAS DE PROTECCIÓN}

A la fecha es posible identificar diversos sistemas, que forman parte de uno más amplio, universal, que es el de $\mathrm{Na}-$ ciones Unidas ${ }^{17}$. Se trata de los sistemas regionales de protección de los derechos humanos, que operan en cada continente, siendo los más desarrollados el europeo y el interamericano ${ }^{18}$.

A los efectos de nuestro estudio, se trabajará sobre la base de ambos sistemas mencionados, elegidos porque, además del europeo, son los que se encuen-

13 Ibid., p. 31.

14 Ibid., p. 33.

15 Los autores utilizan la palabra embeddedness, que no tiene traducción literal al español. Sin embargo, por el contexto en que se utiliza me ha parecido que lo más adecuado es hablar de implementación palabra que refleja más claramente lo que los autores han querido expresar.

16 Goldstein, Judith, op. cit., p. 74.

17 Quesada, Luis Jimena, «El Sistema Europeo de Protección de los Derechos Humanos», Apuntes preparados para la $35^{\circ}$ sesión anual de enseñanza de los derechos humanos impartida por el Instituto Internacional de Derechos Humanos (Cours fondamentaux, Dossier documentaire, Institut International de Droit de L'Homme, $35^{\circ}$ Session Annuelle D’Enseignement, 5-30 Juillet 2004, Strasbourg, France), p. 118.

18 Campusano, Raúl, El Sistema Internacional de Protección a los Derechos Humanos, Santiago, Fundación Friedrich Neumann, Serie Contribuciones, 1994, pp. 12-24. 
tran más desarrollados. El sistema de las Naciones Unidas se considera básico para el estudio, ya que interesa que la protección de las mujeres sea lo más universal posible, por lo cual debiera ser el que se utilice preferentemente $\mathrm{o}$, al menos, ante la falta de otro con alto grado de legalización. La elección del sistema Interamericano se debe a que es el único que cuenta con una convención especial para prevenir y sancionar la violencia contra la mujer ${ }^{19}$.

Finalmente, se considera el sistema que opera en época de conflicto armado, que, si bien forma parte del sistema de protección de las Naciones Unidas, suele tratarse en forma independiente, además de que cuenta con normas propias, con características diferentes ${ }^{20}$.

Legalización del sistema de protección de las Naciones Unidas

\section{Obligación}

Para determinar si el sistema de Naciones Unidas es obligatorio para sus miembros, se deberá hacer un recorrido de las normas existentes en el ámbito in- ternacional y aplicable a la materia, determinando su carácter.

Se refieren específicamente a los derechos de la mujer, la Declaración y Programa de Acción de Viena, derivada de la Conferencia Mundial de Derechos Humanos; la Plataforma de Acción de Beijing, que surgió de la IV Conferencia Mundial sobre la Mujer, realizada en Beijing en 1995; y la Declaración sobre la Eliminación de la Violencia Contra la Mujer. Todos estos instrumentos contienen normas específicas para prevenir la violencia contra la mujer, incluida la violencia sexual ${ }^{21}$. Lamentablemente, estos instrumentos carecen de carácter vinculante, por lo que los gobiernos no se encuentran obligados jurídicamente a cumplir sus disposiciones y solo cuentan con el peso de la opinión pública mundial y la autoridad moral de la comunidad internacional ${ }^{22}$.

\section{Los instrumentos de las Naciones Unidas relativos específicamente a los derechos de la mujer no son vinculantes.} Chilena de Derecho Internacional, Estudios 1995, 1995, pp. 73-91.

20 Naciones Unidas y de sus Organismos Especializados», Apuntes preparados para la $35^{\circ}$ sesión anual de enseñanza de Derechos Humanos impartida por el Instituto Internacional de Derechos Humanos (Cours fondamentaux, Dossier documentaire, Institut International de Droit de L'Homme, $35^{\circ}$ Session Annuelle D'Enseignement, 5-30 Juillet 2004, Strasbourg, France), pp. 44-56.

21 Llanos Mansilla, Hugo, op. cit., pp. 88-91; Informe E/CN.4/2003/75, de la Comisión de Derechos Humanos del Consejo Económico y Social de Naciones Unidas, Integración de los Derechos Humanos de la Mujer y la Perspectiva de Género. La Violencia Contra la Mujer, de 6 de enero de 2003, p. 6.

22 de la Asamblea General», visitada en 27 de octubre de 2004, en <http://www.un.org/spanish/aboutun/ organs/ga/59/background.html>. 


\section{La violencia contra la mujer es consecuencia de la discriminación.}

De este modo, la norma de mayor relevancia resulta ser la Convención sobre la Eliminación de Todas las Formas de Discriminación contra la Mujer, instrumento fundamental, ya que se entiende que la violencia es consecuencia de la discriminación. Además, esta Convención cuenta con un órgano del mismo nombre, que señala expresamente que debe entenderse que la noción de discriminación incluye la violencia basada en el $\mathrm{sexo}^{23}$. Por lo tanto, de alguna manera esta convención también se ocupa de la violencia sexual contra la mujer y como se trata de una convención, en este caso sí posee fuerza obligatoria.

En Naciones Unidas, por tanto, no existe una convención particular que regule el tema de la violencia sexual contra la mujer, aunque sí cuenta con algunas normas vinculantes que se podrían aplicar y con una declaración específica, que por su carácter de tal no obliga a los Estados a respetar sus normas, pero aporta criterios sobre los diversos tipos de violencia a que puede verse enfrentada la mujer, además de pautas respecto de la conducta que cabe a los Estados al respecto $^{24}$.

Por lo señalado, podría sostenerse que en el sistema de Naciones Unidas las normas que protegen a la mujer de la violencia son de nivel medio, ya que si bien no hay falta total de ellas, no regulan el tema en particular y en caso de hacerlo no son vinculantes.

\section{Precisión}

Refiriéndose a las mismas normas antes señaladas, puede observarse que la Declaración y Programa de Acción de Viena, al igual que la Plataforma de Acción de Beijing, solo fijan estándares generales, de modo que si de precisión se trata, no es aquí donde se puede encon$\operatorname{trar}^{25}$.

La Convención sobre la Eliminación de Todas las Formas de Discriminación contra la Mujer, por ser otro su objeto, tampoco es precisa en el tema específico que nos concita. Sin embargo, es la norma principal relativa a la mujer en el ámbito internacional, debido a que es la única que tiene carácter vinculante, por contar con un órgano que verifica su observancia y de algún modo regular la violencia ${ }^{26}$.

23 Guerrero, Elizabeth, Violencia Contra las Mujeres en América Latina y el Caribe Español: 19902000. Balance de una Década, elaborado por Isis Internacional para UNIFEM, Santiago-Chile. 2001, obtenido de la página web <http://www.unifem.org.mx/paginas/documentoselectronicos.asp>, pp. 9-10.

24 Declaración sobre la Eliminación de la Violencia contra la Mujer.

25 Llanos Mansilla, Hugo, op. cit., pp. 88-91; Declaración y Programa de Acción de Viena, de 1993; Lima Malvado, María de la Luz, «La Violencia en Contra de la Mujer en la Agenda Internacional, 1975-1995», Revista Mexicana de Política Exterior, Instituto Matías Romero de Estudios Diplomáticos, Editorial Nueva Época, julio-septiembre, 1995, pp. 130-134.

26 Guerrero, Elizabeth, op. cit., pp. 9-10. 
Por su parte, la Declaración sobre la Eliminación de la Violencia contra la Mujer, instrumento más importante, no solo carece de fuerza vinculante y de órganos encargados de velar por su cumplimiento, sino que no es todo lo precisa que se requiere. Es un avance en el sentido de que define la violencia y detalla actos que la pueden constituir, pero no establece fórmulas claras para evitarla, ni responsabilidades por su violación y, en definitiva, entrega toda la responsabilidad a los Es$\operatorname{tados}^{27}$, quienes como se sabe, no han logrado erradicarla. Por ello, es apremiante realizar mayores esfuerzos.

\section{La aplicabilidad de las normas sobre la violencia contra la mujer dependerá de la labor interpretativa de los órganos competentes.}

De acuerdo con el análisis anterior, no es fácil determinar el nivel de precisión de las normas de las Naciones Unidas aplicables a la violencia sexual contra la mujer: si se consideran únicamente las normas vinculantes, habrá que concluir que, por no referirse específicamente a la violencia sexual contra la mujer, carecen por completo de precisión y dependerá de la labor interpretativa de los órganos competentes determinar si son o no aplicables y establecer los niveles de responsabilidad exigibles. Por el contrario, si incluimos las normas no vinculantes, llegaremos a un nivel de precisión más elevado, aunque todavía se requieren grandes avances, por lo que solo podría atribuírseles un nivel medio.

\section{Delegación}

Dentro del sistema de protección de las Naciones Unidas se puede distinguir una protección convencional y una no convencional. El Alto Comisionado de las Naciones Unidas para los Derechos Humanos forma parte de la segunda, junto con el mecanismo de Relatores Especiales, creado por la Comisión de Derechos Humanos y dependiente de esta ${ }^{28}$. La protección convencional puede ser contenciosa, a cargo de la Corte Internacional de Justicia ${ }^{29}$, o puede consistir en mecanismos de información, o cuasicontenciosos, que están a cargo de los comités creados por los tratados para hacer efectivos los derechos que ellos establecen $^{30}$. De los comités existentes, se podría recurrir principalmente al Comité de Derechos Humanos, creado por el Pacto Internacional de Derechos Civiles y Polí$\operatorname{ticos}^{31} \mathrm{y}$ al Comité para la Eliminación de la Discriminación contra la Mujer (CEDAW).

27 Declaración sobre la Eliminación de la Violencia contra la Mujer.

28 Información obtenida del siguiente sitio web, visitado el 25 de enero de 2005: <http://ohchr.org/ spanish/bodies/chr>.

29 Villán Durán, Carlos, op. cit., pp. 39-40.

30 Ibid., pp. 11-23.

31 Campusano, Raúl, op. cit., p. 18. 


\section{Ninguno de los órganos del sistema de las Naciones Unidas puede interpretar normas 0 resolver conflictos surgidos durante su vigencia.}

Si consideramos la delegación como la facultad de los organismos de interpretar normas, hacer operativas las existentes, dictar nuevas y resolver los conflictos que surjan durante su vigencia ${ }^{32}$, que es como la entienden los autores del modelo que hemos adoptado, tendríamos que concluir que el sistema de las Naciones Unidas no contempla esta dimensión del concepto de legalización, ya que ninguno de sus órganos posee estas facultades, en especial el de solución de controversias, puesto que el único órgano que tiene competencia para hacerlo, la Corte Internacional de Justicia, solo resuelve conflictos entre Estados, en tanto que para nuestro estudio los sujetos interesados son particulares, específicamente las mujeres.

$\mathrm{Si}$, por el contrario, desarticulamos el concepto y lo consideramos con flexibilidad, tal vez podemos ser menos rígidos y encontrar algunos grados de delegación. Por ejemplo, reconocer que la comprenden algunos de los elementos de la labor de la Relatora Especial para la Violencia Contra la Mujer, que recibe denuncias, elabora informes sobre la situación de los países y con ello, de algún modo, interpreta las normas y se pronuncia sobre las di- ficultades de aplicación de las existentes. Si aplicamos entonces el modelo que nos guía, podríamos considerar que sí posee cierto nivel de independencia, alto nivel de acceso y nula implementación, por lo cual alcanzaría un nivel intermedio de delegación.

Si abordamos el análisis desde el punto de vista de los Comités, esto es, los de Derechos Humanos y de Eliminación de la Discriminación contra la Mujer, podríamos decir en primer lugar que no son aplicables, ya que no se ocupan del tema específico de la violencia sexual, en especial el primero, ya que el CEDAW ha declarado que el concepto de discriminación comprende el de violencia, pero como estamos aplicando un criterio flexible, puede considerarse que de alguna manera ellos resuelven conflictos, aunque sin carácter vinculante. El primero de estos Comités ha interpretado las normas por cuyo cumplimiento vela; no así el segundo, que tampoco dicta nuevas normas o emite informes que incluyan fórmulas para hacerlas operativas. Por lo tanto, se cumplen ciertas condiciones para considerar la determinación de su nivel de delegación: la independencia tendrá un nivel elevado, aunque no al máximo, el acceso es mayor en el CEDAW que en el Comité de Derechos Humanos, especialmente por las condiciones de admisibilidad y ambos carecen de implementación. Por todo ello, el grado de delegación no sería tan bajo, pero siempre recordando la flexibilidad otorgada al análisis.

32 Abbott, Kenneth W., Robert O. Keohane, Andrew Moravcsik, Anne-Marie Slaughter y Duncan Snidal, «The Concept of Legalization», en Judith Goldstein et al. (eds.), op. cit., pp. 31-33. 
Legalización del sistema

interamericano de protección

\section{Obligación}

Entre los instrumentos específicos del sistema interamericano encontraremos la Convención para Prevenir, Sancionar y Erradicar la Violencia Contra la Mujer, Convención de Belém do Pará33 ${ }^{33}$ que, dentro de la OEA, es la convención que ha alcanzado el mayor número de ratificaciones, exceptuados Estados Unidos, Canadá y Jamaica ${ }^{34}$. Además, ha sido ratificada sin reservas por parte de los Estados, salvo Bahamas ${ }^{35}$.

\section{En el sistema interamericano, las normas tienen mayor grado de obligatoriedad.}

Por tratarse de una Convención, los Estados se encuentran obligados a respetar sus normas, pero además, al no haber hecho reservas, se encuentran obligados en los mismos términos en que esta fue redactada.

En consecuencia, el grado de obligación del sistema interamericano es bastante elevado, ya que si bien es posible mayor obligatoriedad, por ejemplo, si se hiciera responsable no solo a Estados sino también a los autores de atentados, el nivel actual es bastante apropiado, de modo que lo que ahora resta es hacer que los Estados cumplan la Convención.

\section{Precisión}

El sistema interamericano posee una convención específica respecto de la violencia contra la mujer, la Convención Interamericana para Prevenir, Sancionar y Erradicar la Violencia Contra la Mujer, lo que ya constituye un enorme avance en pro de su protección. Sin embargo, no es todo lo precisa que debiera, puesto que no establece qué entiende por actos que constituyen violencia, no prohíbe ciertos actos sin admitir posibilidad de excusa, o entrega a los Estados solo una pauta de las responsabilidades que les encomien$\mathrm{da}^{36}$. Con esto no se quiere desconocer el valor de contar con el único instrumento de carácter vinculante dentro de los sistemas de protección de la mujer, ni de que el Sistema Interamericano cuente con órganos encargados de verificar su observancia; simplemente se quiere determinar el nivel exacto de precisión, de acuerdo con los parámetros que estamos estudiando y según ellos, aún se puede avanzar mucho más, por lo que concluiremos que

33 Suscrita en 1994.

34 Salvioli, Fabián Omar, «El Sistema Interamericano», Apuntes preparados para la $35^{\circ}$ sesión anual de enseñanza de Derechos Humanos impartida por el Instituto Internacional de Derechos Humanos, (Cours fondamentaux, Dossier documentaire, Institut International de Droit de L'Homme, $35^{\circ}$ Session Annuelle D'Enseignement, 5-30 Juillet 2004, Strasbourg, France), p. 250.

35 Información obtenida de la página web de la Comisión Interamericana de Derechos Humanos, visitada en 3 de marzo de 2005: <http://www.cidh.org/Basicos/Basicos8.htm>.

36 Convención Interamericana para Prevenir, Sancionar y Erradicar la Violencia Contra la Mujer. 
el sistema habrá alcanzado un nivel intermedio.

\section{Delegación}

Para el tratamiento de los casos de derechos humanos, el sistema interamericano tiene una doble estructura: la Comisión Interamericana, que es la puerta de entrada al sistema, y la Corte Interamericana, que cumple funciones interpretativas y contenciosas ${ }^{37}$. La Comisión inicia el examen de un asunto, existiendo actio popularis para someterlo a su conocimiento. Si el Estado no cumple la recomendación de la Comisión, esta puede enviar el caso a la Corte, que adopta un fallo definitivo e inapelable ${ }^{38}$.

El sistema interamericano goza de un grado bastante elevado de delegación, debido a que todos sus componentes, independencia, acceso e implementación muestran niveles altos. Ellos pueden variar, según la composición de la Comisión y de la Corte, así como de la sensibilidad de los miembros por los temas de violencia sexual contra la mujer. Por ello, podemos sostener que las condiciones se encuentran dadas para que la protección funcione. Lamentablemente, no se ha recurrido ante la Corte por transgresión de las normas de la Convención Belém do Pará y específicamente por violencia contra la mujer ${ }^{39}$, por lo tanto es indispensable recurrir al sistema para que este comience a operar.

Legalización del sistema que rige en épocas de conflicto armado

Este sistema es parte de los mecanismos contenciosos con que cuentan las Naciones Unidas, pero por sus características singulares, se realiza un estudio independiente.

\section{Las normas de los Convenios de} Ginebra y sus Protocolos Adicionales apuntan a proteger a la mujer contra la violencia sexual.

\section{Obligación}

Para el análisis de este aspecto de la delegación, el principal instrumento a considerar es el Estatuto de Roma de la Corte Penal Internacional (CPI), pero no se podrá dejar de mencionar los estatutos de los tribunales penales internacionales para Rwanda y para la ex Yugoslavia (TPIR-

37 Salvioli, Fabián Omar, op. cit., pp. 256-258.

38 Medina, Cecilia, «Hacia una Manera más Efectiva de Garantizar que las Mujeres Gocen de sus Derechos Humanos en el Sistema Interamericano», en: Rebecca Cook (ed.), Derechos Humanos de la Mujer. Perspectivas Nacionales e Internacionales, Colombia, Asociación Probienestar de la Familia Colombiana, 1997, pp. 261-262; Medina, Cecilia, «El Sistema Interamericano de promoción y Protección de los Derechos Humanos», apuntes preparados por la autora para sus clases en la Facultad de Derecho de la Universidad de Chile, los que se encuentran a disposición en la biblioteca de dicha facultad, pp. 6-17.

39 Salvioli, Fabián Omar, op. cit., pp. 250-253. 
TPIY), así como las normas del derecho internacional humanitario, de las cuales revisten especial relevancia para este estudio los cuatro Convenios de Ginebra y sus dos Protocolos Adicionales, la mayoría de los cuales no fueron adoptados con el objeto específico de proteger a la mujer contra la violencia sexual, pero contienen normas que en mayor o menor medida apuntan a lograr dicho objeto ${ }^{40}$.

\section{La protección otorgada por los estatutos de los tribunales penales especiales y de la CPI abarca un amplio espectro de atentados de carácter sexual.}

Todos los instrumentos mencionados rigen en épocas de conflicto armado, sea interno o internacional, previa ratificación de los tratados ${ }^{41}$ y siempre que se reúnan los requisitos para su aplicación. Las normas son obligatorias, y en este caso incluso van más allá de la responsabilidad de los Estados, porque también la generan para los individuos ${ }^{42}$.

De este modo, el sistema que rige en épocas de conflicto armado cuenta con el mayor nivel de obligación posible, y se sitúa por encima de los demás sistemas revisados, puesto que no solo obliga a los Estados, sino también a grupos beligerantes e individuos ${ }^{43}$. Además, el tipo de norma es mucho más próxima a los tipos legales, y generan las mayores restricciones posibles dentro de las normas internacionales ${ }^{44}$.

\section{Precisión}

Si bien los Convenios de Ginebra no se refieren en particular a la violencia sexual, sí lo hacen de manera tangencial. Los Protocolos Adicionales, en cambio, brindan una protección más específica a las mujeres ${ }^{45}$. Sin embargo, tampoco establecen lo que entiende por los actos que prohíbe o las formas de protección que brinda a mujeres que han sido víctimas de tales atentados ${ }^{46}$.

Por su parte, los Estatutos de los tribunales penales internacionales para Rwanda y para la ex Yugoslavia, así como el Estatuto de Roma, contemplan una prohibición expresa de los actos de violencia sexual. Los TPI consideran la violación como cri-

Lindsey, Charlotte, Women Facing War, Ginebra, Suiza, CICR, 2001, pp. 57-60.

$\mathrm{Al}$ respecto cabe señalar que la mayor parte de los Estados miembros de la ONU son parte de los Convenios de Ginebra, por lo que es perfectamente posible hablar de derecho internacional universal.

Lirola Delgado, Isabel y Magdalena Martín Martínez, La Corte Penal Internacional. Justicia Versus Impunidad, Barcelona, Editorial Ariel, 2001, pp. 7-17.

Urbina, Julio Jorge, «La Protección de las Personas Civiles en Poder del Enemigo y el Establecimiento de una Jurisdicción Penal Internacional», Revista Internacional de la Cruz Roja, $\mathrm{N}^{\circ}$ 840, diciembre de 2000. Obtenida de la página <http://www.icrc.org/Web/spa>, visitada en 26 de noviembre de 2004.

Abbott, Kenneth W., Roberto O. Keohane, Andrew Moravcsik, Anne-Marie Slaughter y Duncan Snidal, «The Concept of Legalization», en Judith Goldstein et al. (eds.), op. cit., p. 24.

Lindsey, Charlotte, op. cit., p. 58.

Convenios de Ginebra y Protocolos Adicionales. 
men de lesa humanidad y el Estatuto de Roma amplía esta protección a muchos otros actos de violencia sexual, actos que considera crímenes de lesa humanidad, pero también crímenes de guerra ${ }^{47}$.

\section{El sistema de las Naciones Unidas para proteger a la mujer de la violencia sexual no es obligatorio, pero establece los estándares exigibles.}

El análisis de las normas permite apreciar que alcanzan un alto nivel de precisión, especialmente las de los estatutos de los TPI y del Estatuto de Roma, debido a que consideran los diversos actos de violencia sexual, definen varios de ellos y además les otorga igual tratamiento, con lo cual la protección logra abarcar toda la magnitud de estos graves atentados. Además, lo que no ha sido definido lo ha desarrollado la jurisprudencia de los TPI.

\section{Delegación}

Los órganos por analizar serán los TPI y la CPI. Lamentablemente, la Corte Penal Internacional lleva tan poco tiempo de funcionamiento que aún no es posible establecer los parámetros que ha seguido en su actuar, ni la sensibilidad respecto de la violencia que enfrenta la mujer. Por ello, solo se puede hacer el análisis desde lo que «debe ser», de acuerdo con lo que establece su estatuto.

Los tribunales penales internacionales son instituciones ad hoc, creadas por el Consejo de Seguridad de las Naciones Unidas, a las que se les encomendó la represión de determinadas conductas y el castigo de los responsables ${ }^{48}$. En cuanto a la violencia sexual, los tribunales adoptaron posturas bastante proteccionistas: el TPI para Rwanda reconoció que la violación sistemática de mujeres constituía un crimen contra la humanidad e, incluso, una forma de genocidio y el TPI para la ex Yugoslavia consideró que la violación podía considerarse una forma de tortura ${ }^{49}$. Todo lo cual habla muy en favor de la discreción legal y la sensibilidad respecto de la violencia de que puede ser objeto la mujer, lo que favorece la independencia de estas instituciones.

Un análisis de los tribunales penales internacionales especiales revela un grado muy elevado en todos los aspectos de la delegación, esto es, independencia, acceso e implementación ${ }^{50}$.

La Corte Penal Internacional, por su parte, persigue la responsabilidad penal del individuo, de naturaleza diversa a la de carácter reparatorio. Ambas pueden coincidir, especialmente cuando los individuos no actúan como particulares, sino como agentes u órganos del Estado, lo que generaría ambos tipos de responsabilidades,

47 Lindsey, Charlotte, op. cit., pp. 58-59.

48 Urbina, Julio Jorge, op. cit.

49 Ibid., citando los casos Akayesu y Celebici, respectivamente.

50 Keohane, Robert O., Andrew Moravcsik y Anne-Marie Slaughter, «Legalized Dispute Resolution: Interestate and Transnational», en Judith Goldstein et al. (eds.), op. cit., pp. 73-76.

51 Lirola Delgado, Isabel y Magdalena M. Martín Martínez, op. cit., p. 14. 
pero no por ello dejan de ser tipos distintos. ${ }^{51}$

Es así como en el sistema que rige en épocas de conflicto armado, podemos encontrar altos niveles de delegación, puesto que se comprueba la independencia total, que pasa por la selección y estabilidad absolutas respecto de los cargos, así como por altos niveles de discrecionalidad y recursos apropiados para el cumplimiento de su labor. El acceso también es elevado, ya que estará entregado a la investigación del Fiscal, por lo que dependerá de que tome conocimiento, pero no de que la gente pueda o no acceder a el. En cuanto a la implementación, también podemos estimar que alcanza el nivel más alto, puesto que la Corte podrá perseguir a los culpables dondequiera que estén y los Estados tendrían que impugnar directamente la actuación de la Corte, así como de las Naciones Unidas, en caso de proteger a la persona $^{52}$.

\section{ConClusiones}

El análisis realizado ha permitido comprobar el bajo nivel de legalización del sistema de protección de Naciones Unidas, que no cuenta con normas vinculantes específicas respecto de la violencia sexual contra la mujer, por lo que ésta debe recurrir a otras disposiciones en caso de requerir su protección. Sin embargo, cuenta con una declaración sobre la materia, que pese a no ser obligatoria para las par- tes establece los estándares exigibles, lo que ya constituye un avance respecto del pasado, en que ni siquiera se reconocía que la mujer podía ser objeto de ciertos ataques especiales contra sus derechos y que ello requería de una regulación específica.

\section{Existe un bajo nivel de legalización en el sistema de protección de Naciones Unidas pero un alto grado de delegación.}

Lamentablemente, el nivel de delegación de este sistema es bastante bajo, además de ser complejo. No cuenta con un órgano que resuelva controversias entre las partes, ya que solo los Estados pueden recurrir a la Corte Internacional de Justicia. Son diversas las instituciones que realizan las funciones en que consiste la delegación, pero ninguna de ellas las realiza todas, además no tienen la capacidad de vincular a los Estados con sus decisiones y en consecuencia depende de estos el que comprendan los problemas que enfrenta la mujer por su condición de tal y que adopten medidas para resolverlos.

Pese a lo señalado, de todos los aspectos de la legalización, se puede sostener que en el sistema de las Naciones Unidas el más desarrollado es la delegación, puesto que sus órganos han tratado de sortear estas dificultades, incluso arrogándose competencias de las que care-

52 Keohane, Robert O., Andrew Moravcsik y Anne-Marie Slaughter, «Legalized Dispute Resolution: Interestate and Transnational», en Judith Goldstein et al. (eds.), op. cit., pp. 73-76. 
cen, para contribuir a lograr una mayor igualdad de género, indispensable para reducir la violencia que se produce por esta causa.

El sistema interamericano, por el contrario, tiene niveles bastante elevados de legalización. En efecto, es el único que cuenta con una convención específica relativa a la violencia contra la mujer, que regula entre otras la de tipo sexual, que podría ser mucho más precisa y, además, establece la responsabilidad de los particulares, abordando todos los posibles planos de protección. En todo caso, al menos vincula a los Estados, de modo que se les puede obligar a reparar en caso de determinarse su responsabilidad y a modificar sus normas, para adaptarlas a las acordadas.

El sistema interamericano cuenta, además, con los órganos adecuados para desarrollar la labor de delegación, necesaria para alcanzar los altos niveles de legalización de que hablamos. Si bien ellos apuntan a determinar la responsabilidad de los Estados y no de los particulares, su labor ha sido bastante progresista, en el sentido de establecer la responsabilidad de aquellos, aun por actos realizados por los particulares, cuando ha actuado sin la debida diligencia a que se ha obligado mediante convenciones internacionales. También han efectuado un enorme aporte mediante su función interpretativa, lo que puede contribuir a alcanzar mayores niveles de precisión sin necesidad de acordar una nueva convención o un protocolo adicio- nal, cuestión que requiere tantas negociaciones a que muchas veces los Estados no están dispuestos, por lo que en este caso puede bastar con lo alcanzado y no pretender un nivel perfecto de hard law ${ }^{53}$. La Corte Interamericana, finalmente, realiza un novedoso aporte al establecer que, además de la obligación de indemnizar, los Estados tienen obligaciones de hacer, porque podría contribuir a que estos vayan modificando su legislación interna, adoptando medidas preventivas y, por sobre todo, educando a la población, de manera de eliminar gradualmente las causas que conducen a la violencia contra la mujer.

\section{La labor interpretativa de las normas del sistema interamericano de protección de la mujer ha significado un aporte importante.}

Respecto del sistema que opera cuando hay conflicto armado, tal como se ha señalado al examinar el sistema de las $\mathrm{Na}$ ciones Unidas, fue posible comprobar la hipótesis planteada, esto es, el alto nivel alcanzado por todos los aspectos que componen el concepto de legalización, tanto al aplicarlo a la Corte Penal Internacional, como a los tribunales penales internacionales especiales. Es más, en este caso todos los aspectos se encuentran en su máximo nivel, con lo cual nos encontraríamos prácticamente frente a un tipo puro de legalización.

53 Estamos en presencia de hard law cuando existen altos niveles de legalización; si la legalización decrece nos encontraremos, en cambio, ante soft law. 
De esta manera, salvo en el caso del sistema interamericano, las hipótesis fueron comprobadas. Así, corresponde analizar las consecuencias de este hecho.

Una primera conclusión a que podemos llegar es que, al menos en lo que respecta al sistema universal, se observa una carencia de legalización, lo que indica que es urgente desarrollar normas más específicas que protejan a la mujer; que regulen todas las formas de violencia a que puede verse sometida; establecer instituciones propicias que controlen la adecuación de los Estados a esta normativa y que resuelvan, con carácter vinculante, los conflictos que surjan de su incumplimiento. Además, convendría que el sistema no solo persiguiera la responsabilidad de los Estados, sino también la de los perpetradores directos de los actos. De esta manera se contaría con un sistema preventivo y represivo, orientado a lograr la modificación de los patrones existentes y a sancionar la violación de las normas acordadas.

Es cierto que los autores del marco teórico utilizado sostienen, entre otras cosas, que el optar entre hard y soft law depende de los intereses de los Estados, de la cercanía o lejanía de sus posturas en relación con una materia y de su disposición a negociar y no consideran negativo elegir una fórmula más suave, sobre todo si se tiene en cuenta que una posibilidad es no alcanzar ningún tipo de acuerdo. Sin embargo, hay que recordar que basan su análisis en la conveniencia de los Estados, por lo que nuestra conclusión no será necesariamente la misma si lo que deseamos es determinar el mejor mecanismo de protección para la mujer. Efectivamente, puede ser que para los Estados los costos sean importantes, pero para la mujer no contar con normas significa quedar en la indefensión total. Si el país a que pertenece no se preocupa de establecer mecanismos de prevención ni de reparación y tampoco lo hace la comunidad internacional, tendrá que afrontar sola todas las terribles consecuencias de la violencia sexual.

\section{Para la mujer, no contar con normas significa la indefensión total.}

En consecuencia, el sistema universal es claramente insuficiente y es imperioso desarrollarlo en todos los aspectos del concepto de legalización, pero además poniendo énfasis en que los mecanismos deben dirigirse tanto a la prevención -estableciendo medidas que ataquen las causas de la violencia-como a la reparación, que debe ser integral. Por lo tanto, debe dirigirse a resarcir a la víctima de la mejor manera posible, brindándole ayuda psicológica para que pueda enfrentar el trauma que significa ese tipo de agresión, pero también debieran ser capaces de perseguir no solo al Estado, sino también a los perpetradores del acto.

Esta es la conclusión más clara y más acorde con lo esperado. Sin embargo, al final de este trabajo, las demás conclusiones no parecen tan obvias, con lo que quedarán muchas preguntas por resolver en el futuro.

En efecto, una segunda conclusión es que, tal como se postuló, el sistema que 
opera en períodos de conflicto armado se encuentra en el máximo nivel de legalización posible y supera tanto al de Naciones Unidas como al interamericano, por mucho que en este caso la distancia es mínima. Sin embargo cabe preguntarse si es posible sostener que las mujeres se encuentren más protegidas contra la violencia sexual en épocas de conflicto armado.

\section{El sistema de protección ante conflictos armados está en un nivel superior de legalización.}

La comparación se ha efectuado desde el punto de vista de la legalización, a partir de la cual no hay duda de que este sistema se encuentra en un nivel superior; sin embargo, se trata de normas diversas, factor que la perspectiva utilizada no recoge del todo. Así, los sistemas aplicables en tiempos de paz son de tipo preventivo, se orientan a que los Estados vayan adecuando sus normas y modificando su conducta y la de sus actores internos, y en caso de contravención establecen sistemas de reparación, de modo que los Estados comprendan la conducta que se espera de ellos. El sistema interamericano incluso establece obligaciones de hacer para los Estados, precisamente en el sentido de lo establecido por la norma internacional.

El sistema que opera en épocas de conflicto armado actúa ex post, cuando la contravención ya se ha producido, imponiendo penas a los individuos, de acuerdo con su responsabilidad directa en el hecho.
No cuestiona el accionar del Estado.

Una vez comprendido esto, aunque desde la perspectiva de la legalización este último sistema se encuentra más desarrollado, si incorporamos como elemento a considerar el tipo de norma de que se trata, es posible llegar a conclusiones distintas de las esperadas. Al respecto, es posible que la mujer pueda recurrir a la Corte Penal Internacional y encuentre la norma adecuada para el tipo de ataque de que ha sido objeto, pero cabe la duda de si los referidos ataques comenzarán a disminuir, precisamente porque la norma no se dirige a prevenir, sino a castigar conductas ya realizadas. ¿Son extrapolables, entonces, las consecuencias de la legalización a cualquier tipo de sistema?, ¿adecuarán los Estados su accionar durante los conflictos armados por la existencia del Estatuto de Roma?, ¿no se supone, precisamente, que una de las consecuencias de los conflictos es que se produce una violación de las normas vigentes? Si somos positivos, es posible que se produzca un cambio a largo plazo, de cumplirse el efecto que sostiene que el acceso de los actores internos a cortes o instituciones internacionales puede llevarlos a cambiar sus expectativas y su comportamiento. Por tratarse de un cambio de comportamiento, es necesario esperar que transcurra un tiempo considerable antes de que el efecto sea manifiesto, sobre todo considerando que el Estatuto de Roma lleva muy poco tiempo en vigencia.

Finalmente ¿a qué conclusión puede llegarse dado el alto nivel de legalización del sistema interamericano?

En primer lugar, es necesario subra- 
yar que, de acuerdo con las cifras revisadas, en América Latina la tasa de violencia sexual contra la mujer no es menor que en el resto del mundo, ¿puede eso significar que la teoría de la legalización es errónea? o ¿que sus efectos no son los que postulan los autores? Tal vez esa postura sería un poco precipitada y en la práctica las respuestas sean más complejas.

Habrá que realizar un nuevo estudio para determinar si desde 1994, fecha en que se aprobó la Convención de Belém do Pará, los Estados partes han ido adaptando sus normas internas a lo que ella estableció, tratando de cambiar su comportamiento y el de sus nacionales mediante políticas educativas.

Este último punto sugiere, además, que como consecuencia de la normativa internacional, los Estados no solo intentarán cambiar su actuar, sino también el de sus nacionales, que muchas veces se basa en convicciones profundas $\mathrm{y}$, por lo tanto, habrá que trabajar sobre ellas. ¿Se logrará cambiar las convicciones de las personas en una década? Posiblemente se vaya avanzando en este camino, pero si lo que se pretende son cambios tan profundos, tal vez haya que esperar mucho tiempo más para ver los resultados.

Finalmente, nos parece que el camino adoptado es el correcto y que el sistema internacional debiera seguirlo, incorporando elementos del sistema penal internacional, de modo de contar con los mecanismos adecuados, tanto para prevenir como para sancionar a los Estados e individuos, en caso de contravención de las normas.

Pese a lo anterior, hay que tener presente que se trata de un fenómeno que obedece a causas múltiples y por lo tanto no se terminará con él estableciendo medidas en un solo sentido. Probablemente haya que adoptar un grupo de ellas, con visión de conjunto y con el firme propósito de terminar con hechos que afectan de manera tan profunda a una proporción tan importante de la población.

Además, hay que tener presente que la violencia surge como consecuencia de la discriminación contra la mujer, por lo tanto al intentar combatir la primera nunca debe olvidarse la segunda. Es probable que a corto plazo las medidas adecuadas deban dirigirse a luchar contra la violencia; pero a mayor plazo debiera procurarse modificar los patrones socioculturales que consideran a la mujer como un ser de segunda categoría y no como un ser humano digno del mismo respeto que cualquier persona. 